\title{
Quantum critical effects in mean-field glassy systems
}

\author{
Felix Ritort* \\ Institute of Theoretical Physics, University of Amsterdam, Valckenierstraat 65, 1018 XE Amsterdam, The Netherlands
}

(Received 4 December 1996)

\begin{abstract}
We consider the effects of quantum fluctuations in mean-field quantum spin-glass models with pairwise interactions. We examine the nature of the quantum glass transition at zero temperature in a transverse field. In models (such as the random orthogonal model) where the classical phase transition is discontinuous an analysis using the static approximation reveals that the transition becomes continuous at zero temperature. [S0163-1829(97)03417-6]
\end{abstract}

Spin glasses are models which deserve considerable interest. ${ }^{1}$ In these systems the presence of randomness and frustration can yield very rich behavior. In particular, there is much current interest in the behavior of glassy systems in the presence of quantum fluctuations where the nature of the zero-temperature phase transition is driven by the competition between randomness and quantum effects rather than thermal fluctuations. ${ }^{2}$ This makes the order-disorder transition in quantum glasses belong to a new universality class.

Much work has been devoted to the study of mean-field quantum spin-glass models. In particular, attention has been paid to models with a continuous transition in the EdwardsAnderson order parameter. The simplest example in these class of models is the Sherrington-Kirkpatrick (SK) model ${ }^{3}$ in a transverse field. In this system the critical temperature is depressed when the transverse field is switched on and vanishes for a critical value of the field. ${ }^{4-7}$ Analytical work in the quantum SK model reveals that replica symmetry is broken in the quantum glass phase at zero temperature. ${ }^{8}$ This is an indication that quantum fluctuations do not destroy one of the most interesting features in glassy systems, that is, the coexistence of a large number of phases or states.

There has been also much recent interest in the study of classical spin-glass models with a discontinuous transition in the order parameter. These models are characterized by the existence of a dynamical singularity at a temperature above the static transition. ${ }^{9,10}$ Concerning the statical and dynamical behavior these models are very good candidates for describing real glasses. ${ }^{11}$ On the one hand, the statics gives a natural explanation for the existence of a thermodynamic ideal glass transition driven by an entropy collapse. On the other hand, the dynamics of these mean-field models is described by the mode coupling equations introduced to describe relaxational phenomena in glasses. ${ }^{12}$ In mean-field models metastable states have an infinite lifetime, and hence dynamics is frozen at the dynamical singularity well above the static transition temperature. Below the dynamical transition temperature the system gets trapped in states which have larger energy than the equilibrium one. All these features are absent in models with a continuous transition.

The purpose of this paper is the study of models with a discontinuous transition in the presence of quantum fluctuations at zero temperature. The motivation is twofold. Concerning the statics we note that the transition cannot be driven in the quantum case by an entropy collapse. The rea- son is that the entropy vanishes everywhere at zero temperature. Concerning the dynamics we can also expect a quite different behavior from the classical case. In macroscopic quantum systems at $T=0$ the dynamics is governed by the Schrödinger equation which is nondissipative and there is no room for any kind of thermal-activated processes. It could well be that trapping processes in the metastable glassy phase are suppressed in the presence of quantum fluctuation effects.

The main conclusion of this work is that the glassy scenario presented before is indeed suppressed by quantum fluctuations. We will provide a proof for this statement within the static approximation for a general class of exactly solvable models. Later on we will argue why this result should be generally valid beyond that approximation.

The family of models we are interested in are quantum Ising spin glasses with pairwise interactions in the presence of transverse field. These are described by the Hamiltonian

$$
\mathcal{H}=-\sum_{i<j} J_{i j} \sigma_{i}^{z} \sigma_{j}^{z}-\Gamma \sum_{i} \sigma_{i}^{x}
$$

where $\sigma_{i}^{z}, \sigma_{i}^{x}$ are the Pauli spin matrices and $\Gamma$ is the transverse field. The indices $i, j$ run from 1 to $N$ where $N$ is the number of sites. The $J_{i j}$ are the couplings taken from an ensemble of random symmetric matrices. In the case that the $J_{i j}$ are independent Gaussian variables this Hamiltonian reduces to the quantum SK model ${ }^{3}$ in a transverse field. If the $J_{i j}$ are orthogonal matrices, then Eq. (1) reduces to the random orthogonal model ${ }^{13}(\mathrm{ROM})$ in a transverse field. At zero transverse field the models become classical and display quite different behavior. The SK model has a continuous finite-temperature transition without jump in the EdwardsAnderson order parameter $^{1}$ while the ROM presents a strong discontinuous transition where the Edwards-Anderson order parameter jumps to a value close to 1 at the transition temperature. $^{13}$

In order to solve model (1) we apply the Trotter-Suzuki decomposition $^{14}$ and rewrite the Hamiltonian in terms of classical spins with an extra imaginary time dimension,

$$
\mathcal{H}_{\mathrm{eff}}=A \sum_{i<j} J_{i j} \sum_{t} \sigma_{i}^{t} \sigma_{j}^{t}+B \sum_{i t} \sigma_{i}^{t} \sigma_{i}^{t+1}+C,
$$


where the time index $t$ runs from 1 to $M$ and the spins $\sigma_{i}^{t}$ take the values \pm 1 . The constants $A, B$, and $C$ are given by $A=\beta / M ; B=\frac{1}{2} \ln [\operatorname{coth}(\beta \Gamma / M)]$, and $C=(M N /$ $2) \ln \left[\frac{1}{2} \sinh (2 \beta \Gamma / M)\right]$. Now we apply the replica trick and compute the average over the disorder of the replicated partition function,

$$
\bar{Z}_{J}^{n}=\int[d J] \sum_{\left\{\sigma_{i}^{t}\right\}} \exp \left(\sum_{a=1}^{n} \mathcal{H}_{\mathrm{eff}}^{a}\right),
$$

where $\int[d J]$ means integration over the random ensemble of matrices. This integral can be done using known methods in matrix theory. ${ }^{15,13}$ The final result of Eq. (3) can be written in terms of a generating function $G(x)$ which depends on the particular ensemble of $J_{i j}$ couplings via its spectrum of eigenvalues. For the two examples we will consider in this paper we have $G_{\mathrm{SK}}(x)=x^{2} / 2$ (SK model) and $G_{\mathrm{ROM}}(x)=\frac{1}{2}$ $\left.\ln \left(\sqrt{1+4 x^{2}}-1\right) / 2 x^{2}\right]+\frac{1}{2} \sqrt{1+4 x^{2}}-\frac{1}{2}$ (ROM model). From Eq. (3) we get

$$
\bar{Z}_{J}^{n}=\int d Q d \Lambda \exp [-N F(Q, \Lambda)]
$$

where

$$
F(Q, \Lambda)=-\frac{n C}{N}+\frac{1}{M^{2}} \operatorname{Tr}(Q \Lambda)-\frac{1}{2} \operatorname{Tr} G(A Q)-\ln [H(\Lambda)],
$$

with $Q_{a b}^{t t^{\prime}}, \Lambda_{a b}^{t t^{\prime}}$ being the order parameters and the trace $\operatorname{Tr}$ is done over the replica and time indices. The term $H(\Lambda)$ is given by

$$
H(\Lambda)=\sum_{\sigma} \exp \left(\sum_{a b} \frac{1}{M^{2}} \sum_{t t^{\prime}} \Lambda_{a b}^{t t^{\prime}} \sigma_{a}^{t} \sigma_{b}^{t^{\prime}}+B \sum_{a t} \sigma_{a}^{t} \sigma_{a}^{t+1}\right),
$$

and the free energy is obtained by making the analytic continuation $\beta f=\lim _{n \rightarrow 0} F\left(Q^{*} \Lambda^{*}\right) / n$ where $Q^{*}, \Lambda^{*}$ are solutions of the saddle point equations $\Lambda_{a b}^{t t^{\prime}}=\left(A M^{2} /\right.$ 2) $\left[G^{\prime}(A Q)\right]_{a b}^{t t^{\prime}}\left(G^{\prime}\right.$ stands for derivative of $\left.G\right)$ and $Q_{a b}^{t t^{\prime}}=\left\langle\sigma_{a}^{t} \sigma_{b}^{t^{\prime}}\right\rangle$. The average $\langle(\cdot)\rangle$ is done over the effective Hamiltonian in Eq. (6).

The natural solution to this set of equations is to assume that the order parameters $Q_{a b}^{t t^{\prime}}, \Lambda_{a b}^{t t^{\prime}}$ are independent of the time indices when $a \neq b$ but they are only translational time invariant if $a=b$. As previously said, we are interested in investigating the order of the quantum paramagnet-quantum glass transition. To this end we will focus on the study of the phase boundary of the model, leaving aside subtelities concerning the quantum glass phase. To this end, it is enough to consider a general one-step replica-symmetry breaking solution. ${ }^{1}$ We divide the $n$ replicas into $n / m$ boxes $K$ of size $m$ such that $m$ divides $n$. The saddle point solution when $a$ $\neq b$ takes the form $Q_{a b}^{t t^{\prime}}=q ; \Lambda_{a b}^{t t^{\prime}}=\lambda$ if $a, b \in K$ and $Q_{a b}^{t t^{\prime}}=\Lambda_{a b}^{t t^{\prime}}=0 \quad$ otherwise. For $a=b$ we take $Q_{a a}^{t t^{\prime}}$ $=R_{\left|t-t^{\prime}\right|}, \Lambda_{a a}^{t t^{\prime}}=\Lambda_{\left|t-t^{\prime}\right|}$. In terms of the Matsubara frequencies $\omega_{p}=2 \pi p / M$ we define as usual the order paramaters in Matsubara space $\hat{R}_{p}, \hat{\Lambda}_{p}, \hat{\sigma}_{p} \quad$ (for instance,
$\left.\hat{R}_{p}=M^{-1} \sum_{t=0}^{M-1} e^{i \omega_{p} t} R_{t}\right)$. Then, the free energy, Eq. (5), can be written in terms of the $\hat{R}_{p}, \hat{\Lambda}_{p}$ (details will be shown elsewhere).

The main purpose in this paper is to investigate the glassy scenario in the phase boundary which means to determine the order of the static and dynamical transitions. More concretely, this corresponds to determine the value of the Edwards-Anderson parameter at the transition point. Following Ref. 16 we expand the free energy (5) around $m=1$, $f=f_{0}+(m-1) f_{1}+O\left((m-1)^{2}\right)$ and determine the paramagnetic free energy $f_{0}$ and the correction $f_{1}$. We get

$$
\begin{gathered}
\beta f_{0}=-\frac{C}{N}+\sum_{p} \hat{R}_{p} \hat{\Lambda}_{p}^{*}-\frac{1}{2} \sum_{p} G\left(\beta \hat{R}_{p}\right)-I(\hat{\Lambda}), \\
\beta f_{1}=q \lambda-\frac{\beta q}{2} G^{\prime}\left(\beta \hat{R}_{0}\right)+\frac{1}{2} G\left(\beta \hat{R}_{0}\right)-\frac{1}{2} G\left(\beta\left(\hat{R}_{0}-q\right)\right) \\
+I(\hat{\Lambda})-\exp [-I(\hat{\Lambda})] \int d p(x) \Xi(x) \ln [\Xi(x)],
\end{gathered}
$$

where $I(\hat{\Lambda})=\ln [\Xi(\lambda=0)]$ and $\Xi(x)=\sum_{\hat{\sigma}_{p}} \exp \left[\Theta\left(\hat{\sigma}_{p}, x\right)\right]$, with

$$
\Theta\left(\hat{\sigma}_{p}, x\right)=\sum_{p=0}^{M-1}\left(\hat{\Lambda}_{p}+M B e^{-i \omega_{p}}\right)\left|\hat{\sigma}_{p}\right|^{2}+(2 \lambda)^{1 / 2} x \hat{\sigma}_{0}-\lambda \hat{\sigma}_{0}^{2} .
$$

Note that $f_{0}$ does not depend on $q$ and $\lambda$ as expected for the paramagnetic part of the free energy. The static and dynamical transitions can be investigated through the study of the term $f_{1}$ (which plays the role of a potential function in some spin-glass models ${ }^{17}$ ). The static transition appears when the free energy $f$ coincides with the paramagnetic free energy $f_{0}$, i.e., $f_{1}=0$. The dynamical transition is given by the presence of a soft mode above the static transition and is obtained by solving the equation

$$
\left(\frac{\partial^{2} f_{1}}{\partial q^{2}}\right)\left(\frac{\partial^{2} f_{1}}{\partial \lambda^{2}}\right)-\left(\frac{\partial^{2} f_{1}}{\partial q \partial \lambda}\right)^{2}=0 .
$$

The solution to these equations plus the saddle point equations $\partial f_{1} / \partial q=\partial f_{1} / \partial \lambda=0$ yields the critical temperature and the value of the jump of the order parameter $q$ at the transition. In case the dynamical and the static transition coincide it can be shown that $q=\lambda=0$ and the transition is continuous in the order parameter. These three equations are complemented by the saddle point equations for the parameters $\hat{R}_{p}, \hat{\Lambda}_{p}$, i.e., $\partial f_{0} / \partial \hat{R}_{p}=\partial f_{0} / \partial \hat{\Lambda}_{p}=0$.

We now derive a simple expression for the dynamical transition temperature. Equation (10) for the soft mode can be worked out and one finds

$$
\beta^{2} e^{-I(\Lambda)} G^{\prime \prime}\left(\beta\left(\hat{R}_{0}-q\right)\right) \int_{-\infty}^{\infty} d p(x)\left(\left\langle\hat{s}_{0}^{2}\right\rangle-\left\langle\hat{s}_{0}\right\rangle^{2}\right)^{2} \Xi(x)=1,
$$

where the average $\langle(\cdot)\rangle$ is taken over the effective Hamiltonian (9).

All previous results are exact and do not involve any approximation. We now want to show that at zero temperature 
the quantum transition becomes continuous. To find the order of the quantum phase transition we note that $f_{1}$ in Eq. (8) only depends on $\hat{R}_{p}$ in the case $p=0$. In this paper we consider the static approximation ${ }^{4}$ where only the mode $\hat{R}_{0}$ is taken to be nonzero and $\hat{R}_{p}=\hat{\Lambda}_{p}=0$ for $p>0$. This is a crude approximation, the reliability of which will be discussed later on. Putting $R=\hat{R}_{0}, \Lambda=\hat{\Lambda}_{0}$ we find the following saddle point equations for $R, \Lambda, q, \lambda$ :

$$
\begin{gathered}
\Lambda=\frac{\beta}{2} G^{\prime}(\beta R), \quad R=\left\langle\left\langle\frac{\sinh \left[\Xi_{0}(x)\right] x^{2}}{\Xi_{0}(x)}\right\rangle\right\rangle_{0}, \\
\lambda=\frac{\beta}{2}\left[G^{\prime}(\beta R)-G^{\prime}(\beta(R-q)] ;\right. \\
q=\left\langle\left\langle\frac{\left[\int_{-\infty}^{\infty} d p(z) \sinh (T)(b / T)\right]^{2}}{\int_{-\infty}^{\infty} d p(z) \cosh (T)}\right\rangle\right\rangle_{0},
\end{gathered}
$$

where

$$
\langle\langle(\cdot)\rangle\rangle_{0}=\frac{\int_{-\infty}^{\infty} d p(x)(\cdot)}{\int_{-\infty}^{\infty} d p(x) \cosh \left[\Xi_{0}(x)\right]},
$$

with $\Xi_{0}(x)=\left(2 \Lambda x^{2}+\beta^{2} \Gamma^{2}\right)^{1 / 2}, \quad T=\left(b^{2}+\beta^{2} \Gamma^{2}\right)^{1 / 2}$, and $b=[2(\Lambda-\lambda)]^{1 / 2} z+(2 \lambda)^{1 / 2} x$.

Exact expressions are also obtained for the free energies $f_{0}, f_{1}$ and for Eq. (11). This set of equations can be always numerically solved but explicit results can be analytically obtained in the zero-temperature limit. Substituting the solution $\Lambda=u \beta, \lambda=v \beta$ into Eqs. (12) and performing the integrals with the saddle point method we find after some lengthy computations that $u, v$ and the critical field $\Gamma_{c}$ satisfy the equations

$$
\begin{gathered}
u=\frac{1}{2} G^{\prime}\left(\frac{1}{\Gamma_{c}-2 u}\right), \\
v=\frac{1}{2}\left[G^{\prime}\left(\frac{1}{\Gamma_{c}-2 u}\right)-G^{\prime}\left(\frac{1}{\Gamma_{c}-2(u-v)}\right)\right] .
\end{gathered}
$$

It is easy to check that Eqs. (14) and (15) only admit the trivial solution $v=0$. It is also possible to show that in case $v=0$ also $f_{1}=0$. Because $q$ and $R$ vanish with $T$ and the free energy of this solution coincides with the paramagnetic free energy $f_{0}$, we conclude that the transition becomes continuous at zero temperature. In order to determine the critical field $\Gamma_{c}$ we solve Eq. (11) in the $\beta \rightarrow \infty$ limit which yields

$$
\left(\Gamma_{c}-2 u\right)^{-2} G^{\prime \prime}\left(\frac{1}{\Gamma_{c}-2 u}\right)=1 .
$$

Note that $\chi_{0}=\beta \hat{R}_{0}=\left(\Gamma_{c}-2 u\right)^{-1}$ is the main result of this computation from which we can alternatively derive the main Eqs. (14) and (16). The former can be derived from the first of Eq. (12) while the latter can be simply obtained by

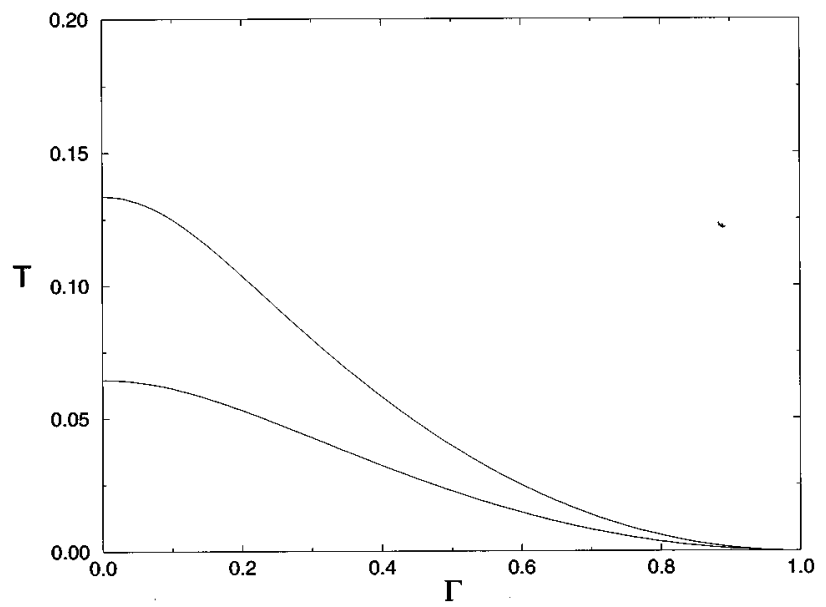

FIG. 1. Phase boundaries $T_{s}(\Gamma)$ (lower line) and $T_{D}(\Gamma)$ (upper line) in the ROM in the static approximation. At zero transverse field $T_{s} \simeq 0.0646, T_{D} \simeq 0.1336$.

deriving Eq. (14) respect to $u$. Solving Eqs. (14) and (16) for the SK model we obtain $u=\frac{1}{2}, \Gamma_{c}=2$, reproducing known results. ${ }^{5,18}$ In the case of the ROM we obtain $u=\frac{1}{2}, \Gamma_{c}=1$. Note that in both models the value of the critical field is given by the maximum eigenvalue of the coupling matrix $J_{i j}$.

To check our analytical computations we have numerically solved Eqs. (12). In Fig. 1 we show the phase boundaries for the dynamical and static transitions in the ROM as a function of the transverse field. Both transition temperatures decrease quadratically as a function of the transverse field, merging into the same point at zero temperature as it should be for a continuous phase transition. In Fig. 2 we show the Edwards-Anderson order parameter $q=\overline{\left\langle\sigma^{z}\right\rangle^{2}}$ in the ROM as a function of $\Gamma$ as we move along the static $\left(q_{S}\right)$ and dynamical $\left(q_{D}\right)$ phase boundaries.

Despite the crudeness of the static approximation we note that still some exact results can be derived in case of a continuous phase transition. Starting from Eq. (11) and putting $(q=\lambda=0)$, Eq. (11), we get

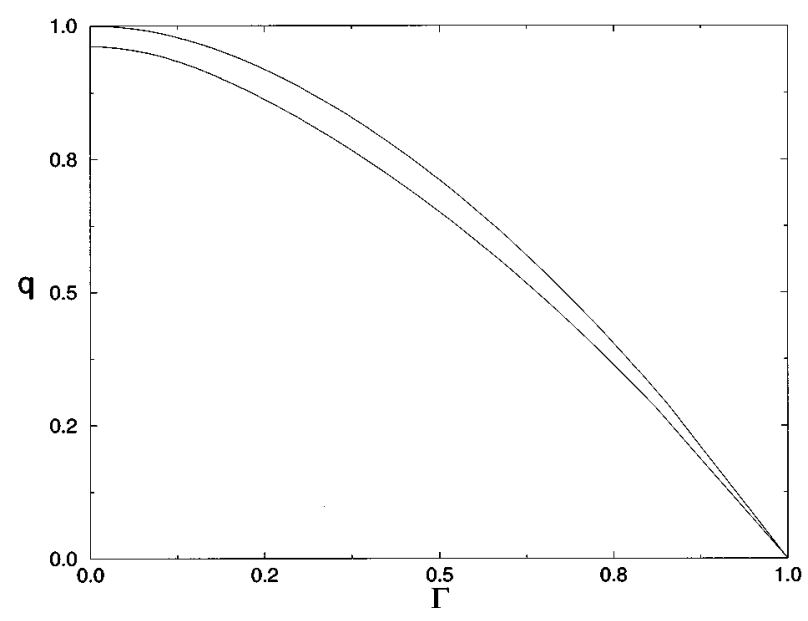

FIG. 2. Edwards-Anderson parameter $q_{s}$ (upper line) and $q_{D}$ (lower line) in the ROM on the static and dynamical phase boundaries boundaries as a function of the transverse field. At zero transverse field $q_{s} \simeq 0.99983, q_{D} \simeq 0.961 . q_{s}$ and $q_{D}$ vanish linearly with $T^{1 / 2}$ at zero temperature. 


$$
\chi_{0}^{2} G^{\prime \prime}\left(\chi_{0}\right)=1,
$$

where $\chi_{0}=\beta\left(\left\langle\hat{s}_{0}^{2}\right\rangle-\left\langle\hat{s}_{0}\right\rangle^{2}\right)=\beta \hat{R}_{0}$ is the longitudinal magnetic susceptibility. This equation can be solved [for a given $G(x)]$ and yields the critical value of $\chi_{0}$. In particular, for the SK model $G_{\mathrm{SK}}^{\prime \prime}(x)=1$ which yields the result $\chi_{0}=1$ in agreement with known results. ${ }^{4}$ In the ROM the only solution to that equation is $\chi_{0}=\infty$. For a continuous transition this implies a divergent susceptibility at the critical field. This is in contrast to the SK model where only the bulk nonlinear susceptibility diverges at the critical field. ${ }^{6} \mathrm{We}$ also note that using a perturbative expansion in powers of $1 / \Gamma$ it would be possible to use Eq. (17) to estimate $\Gamma_{c}$ in the ROM as has been done in the SK case. ${ }^{6}$

To go beyond the static approximation we should consider all the Matsubara modes $\hat{R}_{p}, \hat{\Lambda}_{p}$ in the saddle point equations. The difficulty of this problem is similar to that found in strongly correlated systems where an infinity of parameters has to be computed in a self-consistent way. The static approximation yields inaccurate quantitative results for the thermodynamic properties at low temperatures. Nevertheless, we expect the order of the transition to be correctly predicted. The nature of the transition should not be determined by the decay of the correlation $R_{t}$ in imaginary time but for the particular type of replica symmetry breaking and the Edwards-Anderson parameter at the transition point. Note that the decay of $R_{t}$ for large times is different in the $\mathrm{ROM}$ and in the $S K$ model while the zero-temperature phase transition seems to be continuous in both cases. The reason is that, at zero temperature, $\chi_{0}=\int_{0}^{\infty} d t R_{t}$ which is infinite in the former case but is finite in the latter one $\left[R_{t}\right.$ decays like $t^{-2}$ (Ref. 7)].

Summarizing, we have investigated the glassy behavior in a general class of Ising spin-glass mean-field models with pairwise interactions in the presence of a transverse field. In models with a discontinuous finite-temperature transition we have shown, in the framework of the static approximation, that the transition becomes continuous at $T=0$ and there is no room for a metastable glassy phase. We have argued in favor of this result even beyond the static approximation. According to the mode coupling theory ${ }^{12}$ real glasses are systems characterized by the existence of a dynamical singularity above the glass transition. It is important to note that at finite temperature the dynamical singularity is not associated with any thermodynamic phase transition. Since in quantum systems at zero temperature dynamics and statics are inextricably linked, it is not surprising that this singularity is removed in the only presence of quantum fluctuations. How general this result is for other glassy models remains an interesting open problem. It would be very welcome to extend the present computations beyond the static approximation using recent developed approaches. ${ }^{7}$ It is also worth taking this research further by studying the zero-temperature dynamical transition in quantum $p$-spin-glass models ${ }^{19}$ and Potts glass models. ${ }^{20}$

I thank D. Lancaster, Th. M. Nieuwenhuizen, and F. G. Padilla for discussions and D. Lancaster for a careful reading of the manuscript. This work has been supported by FOM (The Netherlands).
*Electronic address: ritort@phys.uva.nl.

${ }^{1}$ M. Mézard, G. Parisi, and M. A. Virasoro, Spin Glass Theory and Beyond (World Scientific, Singapore, 1987); K. H. Fischer and J. A. Hertz, Spin Glasses (Cambridge University Press, Cambridge, England, 1991).

${ }^{2}$ H. Rieger and A. P. Young, in Coherent Approach to Fluctuations, edited by M. Suzuki and N. Kawashima (World Scientific, Singapore, 1996), p. 161.

${ }^{3}$ D. Sherrington and S. Kirkpatrick, Phys. Rev. Lett. 35, 1792 (1975).

${ }^{4}$ A. J. Bray and M. A. Moore, J. Phys. C. 13, L655 (1980).

${ }^{5}$ K. D. Usadel, Solid State Commun. 58, 629 (1986).

${ }^{6}$ H. Ishii and Y. Yamamoto, J. Phys. C 18, 6225 (1985); 20, 6053 (1987).

${ }^{7}$ J. Miller and D. Huse, Phys. Rev. Lett. 70, 3147 (1993); J. Ye, S. Sachdev, and N. Read, ibid. 70, 4011 (1993).

${ }^{8}$ G. Büttner and K. D. Usadel, Phys. Rev. B 41, 428 (1990); Y. Y. Goldsmichdt and P.Y. Lai, Phys. Rev. Lett. 64, 2467 (1990).

${ }^{9}$ T. R. Kirkpatrick and D. Thirumalai, Phys. Rev. B 36, 5388 (1987); T. R. Kirkpatrick and P. G. Wolyness, ibid. 36, 8552 (1987)
${ }^{10}$ S. Franz and J. Hertz, Phys. Rev. Lett 74, 2114 (1995).

${ }^{11}$ C. A. Angell, Science 267, 1924 (1995).

${ }^{12}$ W. Götze, Liquid, Freezing and the Glass transition, Proceedings of the Les Houches Summer School of Theoretical Physics, City, 1989, edited by J. P. Hansen, D. Levesque, and J. ZinnJustin (North-Holland, Amsterdam, 1989).

${ }^{13}$ E. Marinari, G. Parisi, and F. Ritort, J. Phys. A 27, 7647 (1994).

${ }^{14}$ H. F. Troter, Proc. Am. Math. Soc. 10, 545 (1959); M. Suzuki, Prog. Theor. Phys. 56, 1554 (1976).

${ }^{15}$ C. Itzykson and J-B. Zuber, J. Math. Phys. 21, 411 (1980).

${ }^{16}$ G. Cwilich and T. R. Kirkpatrick, J. Phys A 22, 4971 (1989); E. De Santis, G. Parisi, and F. Ritort, ibid. 28, 3025 (1995).

${ }^{17}$ S. Franz and G. Parisi, J. Phys. (France) I 5, 1401 (1995); R. Monasson, Phys. Rev. Lett. 75, 2847 (1995).

${ }^{18}$ D. Thirumalai, Q. Li, and T. R. Kirkpatrick, J. Phys. A 22, 3339 (1989).

${ }^{19}$ Y. Y. Goldsmichdt, Phys. Rev. B 41, 4858 (1990); V. Dobrosavljevic and D. Thirumalai, J. Phys. A 22, L767 (1990).

${ }^{20}$ T. Senhil and S. N. Majumdar, Phys. Rev. Lett. 76, 3001 (1996). 